\title{
Hops Oil
}

National Cancer Institute

\section{Source}

National Cancer Institute. Hops Oil. NCI Thesaurus. Code C107315.

The essential oil of Humulus lupulus. Hops oil has antimicrobial and antiseptic properties and is also reported to have diuretic and sedative effect. 